\title{
Grossberg-Karshon twisted cubes and hesitant jumping walk avoidance
}

\author{
Eunjeong Lee* \\ Center for Geometry and Physics, Institute for Basic Science (IBS) \\ Pohang 37673, Republic of Korea \\ eunjeong.lee@ibs.re.kr
}

Submitted: Jan 10, 2020; Accepted: July 3, 2020; Published: Aug 21, 2020

(C) The author. Released under the CC BY-ND license (International 4.0).

\begin{abstract}
Let $G$ be a complex simply-laced semisimple algebraic group of rank $r$ and $B$ a Borel subgroup. Let $\mathbf{i} \in[r]^{n}$ be a word and let $\boldsymbol{\ell}=\left(\ell_{1}, \ldots, \ell_{n}\right)$ be a sequence of non-negative integers. Grossberg and Karshon introduced a virtual lattice polytope associated to $\mathbf{i}$ and $\boldsymbol{\ell}$ called a twisted cube, whose lattice points encode the character of a $B$-representation. More precisely, lattice points in the twisted cube, counted with sign according to a certain density function, yield the character of the generalized Demazure module determined by $\mathbf{i}$ and $\boldsymbol{\ell}$. In a recent work, the author and Harada described precisely when the Grossberg-Karshon twisted cube is untwisted, i.e., the twisted cube is a closed convex polytope, in the situation when the integer sequence $\boldsymbol{\ell}$ comes from a weight $\lambda$ for $G$. However, not every integer sequence $\ell$ comes from a weight for $G$. In the present paper, we interpret the untwistedness of Grossberg-Karshon twisted cubes associated with any word $\mathbf{i}$ and any integer sequence $\boldsymbol{\ell}$ using the combinatorics of $\mathbf{i}$ and $\boldsymbol{\ell}$. Indeed, we prove that the Grossberg-Karshon twisted cube is untwisted precisely when $\mathbf{i}$ is hesitant-

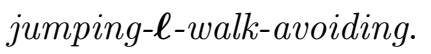

Mathematics Subject Classifications: 20G05, 52B20

\section{Introduction}

Formulating a combinatorial model for a basis of representation provides a fruitful connection between representation theory and algebraic geometry as exhibited by the theory of crystal bases and string polytopes. Kaveh [Kav15] showed that the string polytopes can

*Supported by Basic Science Research Program through the National Research Foundation of Korea (NRF) funded by the Ministry of Science, ICT \& Future Planning (No. 2016R1A2B4010823) and IBSR003-D1 (Institute for Basic Science in Korea). 
be obtained as Newton-Okounkov bodies of the flag variety, and this association is extended to the generalized string polytopes and Bott-Samelson varieties by Fujita [Fuj18]. On the other hand, Grossberg and Karshon [GK94] also provided a combinatorial model called twisted cubes. Considering the lattice points in the twisted cube with signs, the character of the corresponding representation is computed. Note that both generalized string polytopes and twisted cubes are combinatorial models for the same representation.

Twisted cubes are combinatorially much simpler than generalized string polytopes. Indeed, when a twisted cube is closed and convex, then it is combinatorially equivalent to an $n$-cube $[0,1]^{n}$ while the combinatorial structure of a generalized string polytope is not known in general. However, twisted cubes may not be actual polytopes in the sense that they may not be convex nor closed and the intersection of faces may not be a face (cf. [GK94, §2.5 and Figure 1 therein] and Figure 1.1). More precisely, a GrossbergKarshon twisted cube is a pair $(C=C(\mathbf{c}, \ell), \rho)$, where $C$ is a subset of $\mathbb{R}^{n}$ and $\rho$ is a density function whose support is $C$, taking values in $\{ \pm 1\}$. The defining parameters $\mathbf{c}=\left\{c_{j, k}\right\}_{1 \leqslant j<k \leqslant n}$ and $\boldsymbol{\ell}=\left(\ell_{1}, \ldots, \ell_{n}\right)$ are fixed constants with $c_{j, k} \in \mathbb{Z}$ and $\ell_{j} \in \mathbb{R}$.

The main result of this paper concerns twisted cubes obtained from representationtheoretic data. Let $G$ be a complex semisimple algebraic group of rank $r$ and $B$ a Borel subgroup. Indeed, we consider a (not necessarily reduced) word decomposition $\mathbf{i}=\left(i_{1}, \ldots, i_{n}\right) \in[r]^{n}$ of an element $s_{i_{1}} \cdots s_{i_{n}}$ in the Weyl group $W$ of $G$ and non-negative integers $\mathbf{m}=\left(m_{1}, \ldots, m_{n}\right)$. Here, $[r]:=\{1, \ldots, r\}$. In this situation, parameters $\mathbf{c}(\mathbf{i})$ and $\boldsymbol{\ell}(\mathbf{i}, \mathbf{m})$ are associated, and the Grossberg-Karshon twisted cube $(C(\mathbf{c}(\mathbf{i}), \boldsymbol{\ell}(\mathbf{i}, \mathbf{m})), \rho)$ encodes the character of a certain $B$-representation which is the space of holomorphic sections. (See Section 1 for more details.)

In this paper, we present a necessary and sufficient condition on $\mathbf{i}$ and $\boldsymbol{\ell}$ such that the associated Grossberg-Karshon twisted cube is untwisted (see Definition 4), i.e., $C(\mathbf{c}, \ell)$ is a closed convex polytope and the density function is equal to 1 on $C(\mathbf{c}, \ell)$, so that the Grossberg-Karshon character formula is a purely combinatorial positive formula. In other words, there is no minus sign in the formula.

In order to introduce our result, we prepare some terminology (see Section 2 for precise definitions). We say a word $\mathbf{i}=\left(i_{1}, \ldots, i_{n}\right)$ is a jumping walk if for each $2 \leqslant j \leqslant n$, the set $\left\{i_{1}, \ldots, i_{j-1}\right\}$ and an element $i_{j}$ are adjacent in the Dynkin diagram, i.e., the distance $d\left(\left\{i_{1}, \ldots, i_{j-1}\right\}, i_{j}\right)=\min \left\{d\left(i_{k}, i_{j}\right) \mid k=1, \ldots, j-1\right\}$ is one. Here, $d(a, b)$ is the distance of two nodes $a$ and $b$ on the Dynkin diagram. Therefore, to make a jumping walk, one can jump for the next step, but cannot away far. For example, in type $A_{5}$

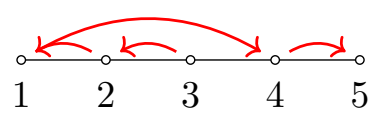

the word $\mathbf{i}=(1,3,2,4)$ is not a jumping walk since $d(1,3)=2$, but $\mathbf{i}=(3,2,1,4,5)$ is a jumping walk. In the above diagram, one can see the jumping walk $(3,2,1,4,5)$. The word $\mathbf{i}=\left(i_{1}, i_{2}, \ldots, i_{n}\right)$ is a hesitant jumping $\boldsymbol{\ell}$-walk if $i_{1}=i_{2}$, the subword $\left(i_{2}, \ldots, i_{n}\right)$ is a jumping walk, and the integers $\ell_{1}, \ell_{2}, \ldots, \ell_{n}$ satisfy an inequality $\ell_{1}-\ell_{2}<\ell_{2}+\cdots+\ell_{n}$. Finally, we say that $\mathbf{i}$ is hesitant-jumping- $\boldsymbol{\ell}$-walk-avoiding if there is no subword $\mathbf{j}=$ 
$\left(i_{j_{0}}, i_{j_{1}}, \ldots, i_{j_{s}}\right)$ of $\mathbf{i}$ which is a hesitant jumping $\left(\ell_{j_{0}}, \ell_{j_{1}}, \ldots, \ell_{j_{s}}\right)$-walk. Now we state our main theorem.

Theorem 1 (Theorem 18). Let $G$ be a complex simply-laced semisimple algebraic group of rank $r$. Let $\mathbf{i}=\left(i_{1}, \ldots, i_{n}\right) \in[r]^{n}$ and $\boldsymbol{\ell}=\left(\ell_{1}, \ldots, \ell_{n}\right) \in \mathbb{Z}_{\geqslant 0}^{n}$. Then the corresponding Grossberg-Karshon twisted cube is untwisted if and only if $\mathbf{i}$ is hesitant-jumping- $\boldsymbol{\ell}$-walkavoiding.

We note that if we consider a simpler situation such that the pair $(\mathbf{i}, \mathbf{m})$ satisfies a condition that $m_{j} \neq 0$ only if $i_{j^{\prime}} \neq i_{j}$ for any $j<j^{\prime} \leqslant n$, then the untwistedness of the corresponding Grossberg-Karshon twisted cube can be detected using hesitant $\lambda$-walk avoidance by Harada and the author [HL15]. Here, $\lambda$ encodes the data of nonzero $m_{j}$ 's. However, not every pair $(\mathbf{i}, \mathbf{m})$ satisfies the condition usually. Since our main result can be applied to any pair $(\mathbf{i}, \mathbf{m})$, the present result is more powerful than the previous one [HL15] (see Remark 10 and Corollary 20).

Additionally, for given $\mathbf{i} \in[r]^{n}$ and $\mathbf{m}$, with an appropriate choice of a valuation $\nu$, Harada and Yang [HY16] constructed a Newton-Okounkov body $\Delta=\Delta(\mathbf{i}, \mathbf{m}, \nu)$, which is a closed convex polytope. They proved that when the twisted cube $(C(\mathbf{c}(\mathbf{i}), \boldsymbol{\ell}(\mathbf{i}, \mathbf{m})), \rho)$ is untwisted, then the Newton-Okounkov body $\Delta$ and the twisted cube $C(\mathbf{c}(\mathbf{i}), \boldsymbol{\ell}(\mathbf{i}, \mathbf{m}))$ are the same (up to a certain coordinate change). Our result presents a sufficient condition on $\mathbf{i}$ and $\mathbf{m}$ such that the Newton-Okounkov body $\Delta$ coincides with the twisted cube.

This paper is organized as follows. In Section 1, we recall the necessary definitions and establish terminology and notation. In Section 2, we introduce the notions of jumping walks, hesitant jumping walks, and hesitant-jumping- $\boldsymbol{\ell}$-walk-avoidance. Using this terminology we then make the statement of our main result, which is that untwistedness is equivalent to hesitant-jumping- $\boldsymbol{\ell}$-walk-avoidance. The proof of the main result occupies Section 3.

\section{Background on Grossberg-Karshon twisted cubes}

We begin by recalling the definition of twisted cubes introduced by Grossberg and Karshon [GK94, §2.5]. Let $n$ be a fixed positive integer. A twisted cube is defined to be a pair $(C(\mathbf{c}, \boldsymbol{\ell}), \rho)$, where $C(\mathbf{c}, \boldsymbol{\ell})$ is a subset of $\mathbb{R}^{n}$ and $\rho: \mathbb{R}^{n} \rightarrow \mathbb{R}$ is a density function with support equal to $C(\mathbf{c}, \boldsymbol{\ell})$. Here, $\mathbf{c}=\left\{c_{j, k}\right\}_{1 \leqslant j<k \leqslant n}$ and $\boldsymbol{\ell}=\left(\ell_{1}, \ell_{2}, \ldots, \ell_{n}\right)$ are fixed integers. In order to simplify the notation in what follows, we define the following functions on $\mathbb{R}^{n}$ :

$$
\begin{aligned}
& A_{n}(x)=A_{n}\left(x_{1}, \ldots, x_{n}\right)=\ell_{n} \\
& A_{j}(x)=A_{j}\left(x_{1}, \ldots, x_{n}\right)=\ell_{j}-\sum_{k>j} c_{j, k} x_{k} \quad \text { for all } 1 \leqslant j \leqslant n-1 .
\end{aligned}
$$

We also define a function $\operatorname{sgn}: \mathbb{R} \rightarrow\{ \pm 1\}$ by $\operatorname{sgn}(x)=1$ for $x<0$ and $\operatorname{sgn}(x)=-1$ for $x \geqslant 0$.

We now give the definition of twisted cubes. 
Definition 2. Let $n, \mathbf{c}, \boldsymbol{\ell}$ and $A_{j}$ be as above. Let $C(\mathbf{c}, \boldsymbol{\ell})$ denote the following subset of $\mathbb{R}^{n}$ :

$$
\begin{aligned}
C(\mathbf{c}, \ell):=\{x= & \left(x_{1}, \ldots, x_{n}\right) \in \mathbb{R}^{n} \mid \\
& \left.A_{j}(x)<x_{j}<0 \text { or } 0 \leqslant x_{j} \leqslant A_{j}(x) \quad \text { for } 1 \leqslant j \leqslant n\right\} .
\end{aligned}
$$

Moreover, we define a density function $\rho: \mathbb{R}^{n} \rightarrow \mathbb{R}$ by

$$
\rho(x)= \begin{cases}(-1)^{n} \prod_{k=1}^{n} \operatorname{sgn}\left(x_{k}\right) & \text { if } x \in C(\mathbf{c}, \boldsymbol{\ell}), \\ 0 & \text { else. }\end{cases}
$$

Obviously, the support $\operatorname{supp}(\rho)$ of the density function $\rho$ is $C(\mathbf{c}, \boldsymbol{\ell})$. We call the pair $(C(\mathbf{c}, \boldsymbol{\ell}), \rho)$ the twisted cube associated to $\mathbf{c}$ and $\boldsymbol{\ell}$.

A twisted cube may not be combinatorially equivalent to a cube $[0,1]^{n}$ in the standard sense. In particular, the set $C(\mathbf{c}, \boldsymbol{\ell})$ may be neither convex nor closed, as the following example shows. See also the discussion in [GK94, §2.5].

Example 3. Let $n=2, \boldsymbol{\ell}=\left(\ell_{1}=2, \ell_{2}=3\right)$ and $\mathbf{c}=\left\{c_{1,2}=1\right\}$. Then $C:=C(\mathbf{c}, \boldsymbol{\ell})$ consists of points $\left(x_{1}, x_{2}\right) \in \mathbb{R}^{2}$ satisfying

$$
\begin{aligned}
0 & \leqslant x_{2} \leqslant 3, \\
2-x_{2}<x_{1}<0 \text { or } 0 & \leqslant x_{1} \leqslant 2-x_{2} .
\end{aligned}
$$

See Figure 1.1 for the set $C$. The value of the density function $\rho$ is recorded within each region.

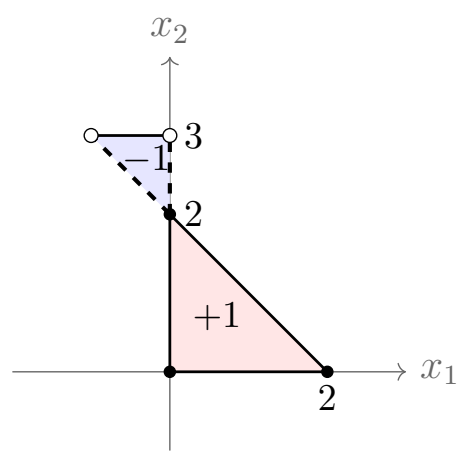

Figure 2.1: A twisted cube.

Note that the subset $C$ does not contain the points $\left\{\left(0, x_{2}\right) \mid 2<x_{2}<3\right\}$ and the points $\left\{\left(x_{1}, x_{2}\right) \mid 2<x_{2}<3\right.$ and $\left.x_{1}=2-x_{2}\right\}$, so $C$ is neither closed nor convex.

As mentioned in the introduction, the primary goal of this manuscript is to give necessary and sufficient conditions for the untwistedness of the twisted cube, in terms of the combinatorics of the defining parameters. The following makes this notion precise. 
Definition 4 (cf. [HY15, Definition 2.2]). We say that the Grossberg-Karshon twisted cube $(C=C(\mathbf{c}, \ell), \rho)$ is untwisted if $C$ is a closed convex polytope, and the density function $\rho$ is equal to 1 on $C$ and 0 elsewhere.

The main result of [HY15] characterizes the untwistedness of the Grossberg-Karshon twisted cube in terms of the basepoint-freeness of a certain toric divisor on a toric variety constructed from the data of $\mathbf{c}$ and $\boldsymbol{\ell}$. In particular, their result can be stated in terms of the Cartier data $\left\{m_{\sigma}\right\}$ associated to the divisor on the toric variety. Before reviewing the relevant result from [HY15], we introduce some terminology.

Let $\left\{e_{1}^{+}, \ldots, e_{n}^{+}\right\}$be the standard basis of $\mathbb{R}^{n}$. For $\sigma=\left(\sigma_{1}, \ldots, \sigma_{n}\right) \in\{+,-\}^{n}$, define $m_{\sigma}=\left(m_{\sigma, 1}, \ldots, m_{\sigma, n}\right)=\sum_{j=1}^{n} m_{\sigma, j} e_{j}^{+} \in \mathbb{Z}^{n}$ as follows.

$$
m_{\sigma, j}= \begin{cases}0 & \text { if } \sigma_{j}=+, \\ A_{j}(\underbrace{0, \ldots, 0}_{j}, m_{\sigma, j+1}, \ldots, m_{\sigma, n}) & \text { if } \sigma_{j}=-.\end{cases}
$$

Here, $A_{j}(x)$ are the functions in (1.1). With this notation, we recall the following.

Theorem 5 (cf. [HY15, Proposition 2.1]). Let $n, \mathbf{c}$ and $\boldsymbol{\ell}$ be as above and let $(C(\mathbf{c}, \boldsymbol{\ell}), \rho)$ denote the corresponding Grossberg-Karshon twisted cube. Then $(C(\mathbf{c}, \boldsymbol{\ell}), \rho)$ is untwisted if and only if $m_{\sigma, j} \geqslant 0$ for all $\sigma \in\{+,-\}^{n}$ and for all $j$ with $1 \leqslant j \leqslant n$.

Remark 6 (cf. [HY15, §1.2]). Define

$$
e_{j}^{-}:=-e_{j}^{+}-\sum_{k>j} c_{j, k} e_{k}^{+} \quad \text { for } 1 \leqslant j \leqslant n .
$$

Let $\Sigma(\mathbf{c})$ be the fan consisting of maximal cones generated by $\left\{e_{j}^{\sigma_{j}} \mid 1 \leqslant j \leqslant n\right\}$ for each $\sigma=\left(\sigma_{1}, \ldots, \sigma_{n}\right) \in\{+,-\}^{n}$. The toric variety $X(\Sigma(\mathbf{c}))$ constructed by the fan $\Sigma(\mathbf{c})$ is called a Bott manifold. For the torus-invariant divisor

$$
D(\mathbf{c}, \ell):=\sum_{j=1}^{n} \ell_{j} D_{e_{j}^{-}}
$$

the Cartier data for $D(\mathbf{c}, \boldsymbol{\ell})$ is the same as $\left\{m_{\sigma}\right\}$. Here, $D_{e_{j}^{-}}$denotes the torus-invariant divisor corresponding to the ray spanned by $e_{j}^{-}$for $1 \leqslant j \leqslant n$.

Recall that we will study the case when the defining parameters for the GrossbergKarshon twisted cube arise from certain representation-theoretic data. We now briefly describe how to derive the $\mathbf{c}$ and $\boldsymbol{\ell}$ in this case.

Following the setting in [GK94], let $G$ be a complex semisimple linear algebraic group of rank $r$. Choose a Cartan subgroup $H \subset G$, and let $\mathfrak{g}=\mathfrak{h} \oplus \bigoplus_{\alpha} \mathfrak{g}_{\alpha}$ be the decomposition into root spaces. We choose a set $\Delta^{+}$of positive roots, and let $B$ be the Borel subgroup whose Lie algebra is $\mathfrak{h} \oplus \bigoplus_{\alpha \in \Delta^{+}} \mathfrak{g}_{-\alpha}$. Let $\left\{\alpha_{1}, \ldots, \alpha_{r}\right\}$ denote the simple roots, $\left\{\alpha_{1}^{\vee}, \ldots, \alpha_{r}^{\vee}\right\}$ the simple coroots, and $\left\{\varpi_{1}, \ldots, \varpi_{r}\right\}$ the fundamental weights. Note that 
fundamental weights are characterized by the relation $\left\langle\varpi_{i}, \alpha_{j}^{\vee}\right\rangle=\delta_{i j}$. Let $W$ be the Weyl group of $G$ and $s_{\alpha} \in W$ denote the simple reflection in $W$ corresponding to the root $\alpha$. For simplicity, we denote $s_{i}$ for the reflection $s_{\alpha_{i}}$ corresponding to the simple root $\alpha_{i}$.

Let $\mathbf{i}=\left(i_{1}, \ldots, i_{n}\right)$ be a sequence of elements in $[r]$ and $\mathbf{m}=\left(m_{1}, \ldots, m_{n}\right) \in \mathbb{Z}_{\geqslant 0}^{n}$. Then $\mathbf{i}$ corresponds to a decomposition of an element $w=s_{i_{1}} s_{i_{2}} \cdots s_{i_{n}}$ in $W$ which is not necessarily reduced. For such $\mathbf{i}$ and $\mathbf{m}$, we define constants $\mathbf{c}(\mathbf{i})=\left\{c_{j, k}\right\}_{1 \leqslant j<k \leqslant n}$ and $\boldsymbol{\ell}(\mathbf{i}, \mathbf{m})=\left(\ell_{1}, \ldots, \ell_{n}\right)$ by the formulas in $[\mathrm{GK} 94, \S 3.7]$

$$
\begin{aligned}
c_{j, k} & =\left\langle\alpha_{i_{k}}, \alpha_{i_{j}}^{\vee}\right\rangle \quad \text { for } 1 \leqslant j<k \leqslant n, \\
\ell_{j} & =\left\langle m_{j} \varpi_{i_{j}}+\cdots+m_{n} \varpi_{i_{n}}, \alpha_{i_{j}}^{\vee}\right\rangle \quad \text { for } 1 \leqslant j \leqslant n .
\end{aligned}
$$

Note that the constants $c_{j, k}$ are Cartan integers of $G$. The following example illustrates these definitions.

Example 7. Consider $G=\operatorname{SL}(3, \mathbb{C})$ with simple roots $\left\{\alpha_{1}, \alpha_{2}\right\}$. Let $\mathbf{i}=(1,2,1)$ and $\mathbf{m}=(1,1,1)$. Then we have

$$
c_{1,2}=\left\langle\alpha_{2}, \alpha_{1}^{\vee}\right\rangle=-1, \quad c_{1,3}=\left\langle\alpha_{1}, \alpha_{1}^{\vee}\right\rangle=2, \quad c_{2,3}=\left\langle\alpha_{1}, \alpha_{2}^{\vee}\right\rangle=-1,
$$

and

$$
\begin{aligned}
& \ell_{1}=\left\langle\varpi_{1}+\varpi_{2}+\varpi_{1}, \alpha_{1}^{\vee}\right\rangle=2, \\
& \ell_{2}=\left\langle\varpi_{2}+\varpi_{1}, \alpha_{2}^{\vee}\right\rangle=1, \\
& \ell_{3}=\left\langle\varpi_{1}, \alpha_{1}^{\vee}\right\rangle=1 .
\end{aligned}
$$

Example 8. Consider $G=\mathrm{SO}(8)$ with simple roots $\left\{\alpha_{1}, \ldots, \alpha_{4}\right\}$. See Table 1 for the numbering on simple roots. Let $\mathbf{i}=(1,2,3,2,4)$ and $\mathbf{m}=(2,1,3,1,1)$. Then the integers $c_{j, k}$ and $\boldsymbol{\ell}$ are given by

$$
\left(c_{j, k}\right)=\left(\begin{array}{ccccc}
0 & -1 & 0 & -1 & 0 \\
0 & 0 & -1 & 2 & -1 \\
0 & 0 & 0 & -1 & 0 \\
0 & 0 & 0 & 0 & -1 \\
0 & 0 & 0 & 0 & 0
\end{array}\right), \quad \boldsymbol{\ell}=(2,2,3,1,1) .
$$

Here, we set $c_{j, k}=0$ if $j \geqslant k$.

Geometrically, a word $\mathbf{i}=\left(i_{1}, \ldots, i_{n}\right)$ and the integer vector $\mathbf{m}$ define a Bott-Samelson variety $Z_{\mathbf{i}}$ and a line bundle $\mathcal{L}_{\mathbf{i}, \mathbf{m}}$ on it. More precisely, the Bott-Samelson variety $Z_{\mathbf{i}}$ is defined to be the quotient

$$
Z_{\mathbf{i}}=\left(P_{i_{1}} \times \cdots \times P_{i_{n}}\right) / B^{n},
$$

where $P_{i}$ is the parabolic subgroup associated with the simple root $\alpha_{i}$, i.e., its Lie algebra is $\mathfrak{g}_{\alpha_{i}} \oplus \operatorname{Lie}(B)$, and the right action of $B^{n}$ on $P_{i_{1}} \times \cdots \times P_{i_{n}}$ is given by

$$
\left(p_{1}, \ldots, p_{n}\right) \cdot\left(b_{1}, \ldots, b_{n}\right)=\left(p_{1} b_{1}, b_{1}^{-1} p_{2} b_{2}, \ldots, b_{n-1}^{-1} p_{n} b_{n}\right) .
$$


The multiplication map $\left(p_{1}, \ldots, p_{n}\right) \mapsto p_{1} \cdots p_{n}$ yields a well-defined morphism

$$
\mu: Z_{\mathbf{i}} \rightarrow G / B
$$

The integer vector $\mathbf{m}=\left(m_{1}, \ldots, m_{n}\right)$ defines the line bundle $\mathcal{L}_{\mathbf{i}, \mathbf{m}} \rightarrow Z_{\mathbf{i}}$

$$
\mathcal{L}_{\mathbf{i}, \mathbf{m}}=\left(P_{i_{1}} \times \cdots \times P_{i_{n}} \times \mathbb{C}\right) / B^{n},
$$

where the right action of $B^{n}$ on $P_{i_{1}} \times \cdots \times P_{i_{n}} \times \mathbb{C}$ is defined by

$$
\begin{aligned}
& \left(p_{1}, \ldots, p_{n}, v\right) \cdot\left(b_{1}, \ldots, b_{n}\right) \\
& \quad=\left(p_{1} b_{1}, b_{1}^{-1} p_{2} b_{2}, \ldots, b_{n-1}^{-1} p_{n} b_{n},\left(m_{1} \varpi_{i_{1}}\right)\left(b_{1}\right) \cdots\left(m_{n} \varpi_{i_{n}}\right)\left(b_{n}\right) v\right) .
\end{aligned}
$$

In this setting, the Bott-Samelson variety $Z_{\mathbf{i}}$ determined by the sequence $\mathbf{i}$ has a toric degeneration to the toric variety $X(\Sigma(\mathbf{c}(\mathbf{i}))$ ) (see [Pas10, §1] and [GK94]). Moreover, the line bundle $\mathcal{L}_{\mathbf{i}, \mathbf{m}}$ over the Bott-Samelson variety $Z_{\mathbf{i}}$ degenerates into the line bundle over the Bott manifold $X(\Sigma(\mathbf{c}(\mathbf{i})))$. In particular, the degeneration of the line bundle $\mathcal{L}_{\mathbf{i}, \mathbf{m}}$ over $X(\Sigma(\mathbf{c}(\mathbf{i})))$ is given by the divisor $D(\mathbf{c}(\mathbf{i}), \boldsymbol{\ell}(\mathbf{i}, \mathbf{m}))$.

Remark 9. Grossberg and Karshon [GK94] constructed a one-parameter family of complex structures on a Bott-Samelson variety which makes the Bott-Samelson variety into a Bott manifold. This degeneration of complex structures can be interpreted as the toric degeneration of a Bott-Samelson variety to a Bott manifold by Pasquier [Pas10]. Indeed, there is a flat family $\mathfrak{X}$ over $\mathbb{C}$ such that $\mathfrak{X}(t)$ is isomorphic to the Bott-Samelson variety for all $t \in \mathbb{C} \backslash\{0\}$ and $\mathfrak{X}(0)$ is the Bott manifold. Here, $\mathfrak{X}(t)$ denotes the fiber over $t$. This connection is generalized to flag Bott-Samelson varieties and flag Bott manifolds in [FLS].

The set $H^{0}\left(Z_{\mathbf{i}}, \mathcal{L}_{\mathbf{i}, \mathbf{m}}\right)$ of holomorphic sections possesses a $B$-representation structure, and indeed its dual is a generalized Demazure module. Moreover, the ordinary Demazure module can be obtained in this way. Indeed, suppose that $\mathbf{i}=\left(i_{1}, \ldots, i_{n}\right) \in[r]^{n}$ is a reduced decomposition of an element $w$ in the Weyl group $W$, i.e., $w=s_{i_{1}} \cdots s_{i_{n}}$ and $\ell(w)=n$. Then a dominant integral weight ${ }^{1} \lambda=\sum_{i=1}^{r} \lambda_{i} \varpi_{i}$ gives a line bundle $\mathcal{L}_{\lambda}$ on the flag variety $G / B$, so that on the Schubert variety $X(w):=\overline{B w B / B} \subset G / B$. Define $\mathbf{m}=\left(m_{1}, \ldots, m_{n}\right) \in \mathbb{Z}_{\geqslant 0}^{n}$ by

$$
m_{j}= \begin{cases}\lambda_{i_{j}} & \text { if } i_{j^{\prime}} \neq i_{j} \text { for any } j<j^{\prime} \leqslant n \\ 0 & \text { otherwise }\end{cases}
$$

for $1 \leqslant j \leqslant n$. Then, we have that

$$
\mu^{*} \mathcal{L}_{\lambda}=\mathcal{L}_{\mathbf{i}, \mathbf{m}}
$$

and the morphism $\mu$ induces an isomorphism of $B$-modules

$$
H^{0}\left(X(w), \mathcal{L}_{\lambda}\right) \simeq H^{0}\left(Z_{\mathbf{i}}, \mu^{*} \mathcal{L}_{\lambda}\right) \simeq \mathbb{C}_{-\lambda^{\prime}} \otimes H^{0}\left(Z_{\mathbf{i}}, \mathcal{L}_{\mathbf{i}, \mathbf{m}}\right),
$$

\footnotetext{
${ }^{1} \mathrm{~A}$ weight $\lambda=\sum_{i=1}^{r} \lambda_{i} \varpi_{i}$ is dominant integral if $\lambda_{i} \in \mathbb{Z}_{\geqslant 0}$ for all $i$.
} 
where $\lambda^{\prime}=\sum_{i \in[r] \backslash\left\{i_{1}, \ldots, i_{n}\right\}} \lambda_{i} \varpi_{i}$. We note that the relation (1.5) and the second isomorphism of $B$-modules in (1.6) hold even when $\mathbf{i}$ is not reduced. See [Fuj18, Section 2] and references therein for more details on generalized Demazure modules.

Remark 10. Since the Picard number of $Z_{\mathbf{i}}$ is $n$ and that of $G / B$ is $r$, not every line bundle over $Z_{\mathbf{i}}$ can be obtained from $\mathcal{L}_{\lambda}$. For example, let $G=\operatorname{SL}(3, \mathbb{C})$ and $\mathbf{i}=(1,2,1)$. Consider a morphism $\mu: Z_{\mathbf{i}} \rightarrow G / B$ and a dominant weight $\lambda=\lambda_{1} \varpi_{1}+\lambda_{2} \varpi_{2}$. Using (1.5), we obtain

$$
\mu^{*} \mathcal{L}_{\lambda}=\mathcal{L}_{(1,2,1),\left(0, \lambda_{2}, \lambda_{1}\right)} .
$$

For this reason, any line bundle $\mathcal{L}_{(1,2,1),\left(m_{1}, m_{2}, m_{3}\right)}$ with $m_{1} \neq 0$ cannot be expressed as the form $\mu^{*} \mathcal{L}_{\lambda}$.

As a consequence of equations (1.3) and (1.4), we obtain the following result which will be used later:

Lemma 11. Let $\mathbf{i}=\left(i_{1}, \ldots, i_{n}\right) \in[r]^{n}$ and $\lambda=\sum_{i=1}^{r} \lambda_{i} \varpi_{i}$ a dominant weight. Suppose that $\mathbf{m}$ is given by (1.4). Then the constant $\boldsymbol{\ell}(\mathbf{i}, \mathbf{m})=\left(\ell_{1}, \ldots, \ell_{n}\right)$ is given by the formula

$$
\ell_{j}=\lambda_{i_{j}} \quad \text { for } 1 \leqslant j \leqslant n .
$$

Proof. From (1.3) and (1.4), we get

$$
\begin{aligned}
\ell_{j} & =\left\langle m_{j} \varpi_{i_{j}}+\cdots+m_{n} \varpi_{i_{n}}, \alpha_{i_{j}}^{\vee}\right\rangle \\
& =\sum_{\substack{j^{\prime} \geqslant j, i_{j}=i_{j^{\prime}}}} m_{j^{\prime}} \\
& =\lambda_{i_{j}} \quad(\text { by }(1.4)) .
\end{aligned}
$$

This proves the lemma.

As mentioned in the introduction, Grossberg and Karshon derived a Demazure-type character formula for the $B$-representation $H^{0}\left(Z_{\mathbf{i}}, \mathcal{L}_{\mathbf{i}, \mathbf{m}}\right)$ corresponding to $\mathbf{i}$ and $\mathbf{m}$, expressed as a sum over the lattice points $\mathbb{Z}^{n} \cap C(\mathbf{c}, \boldsymbol{\ell})$ in the Grossberg-Karshon twisted cube $(C(\mathbf{c}, \ell), \rho)$ (see [GK94, Theorems 5 and 6$]$ ). The lattice points appear with a plus or minus sign according with the density function $\rho$. Accordingly, their formula is a positive formula if $\rho$ is equal to 1 on all of $C(\mathbf{c}, \boldsymbol{\ell})$. From a point of view of representation theory, it is therefore of interest to determine conditions on the integer vector $\boldsymbol{\ell}=\left(\ell_{1}, \ldots, \ell_{n}\right)$ and the word decomposition $\mathbf{i}=\left(i_{1}, \ldots, i_{n}\right)$ such that the associated Grossberg-Karshon twisted cube is in fact untwisted.

\section{Diagram jumping walks, hesitant jumping walk avoidance, and the statement of the main theorem}

From now on, we assume that the group $G$ is simply-laced, i.e., the Dynkin diagram of $G$ only contains simple links. Accordingly, the Lie algebra of $G$ is a direct sum of simple Lie 


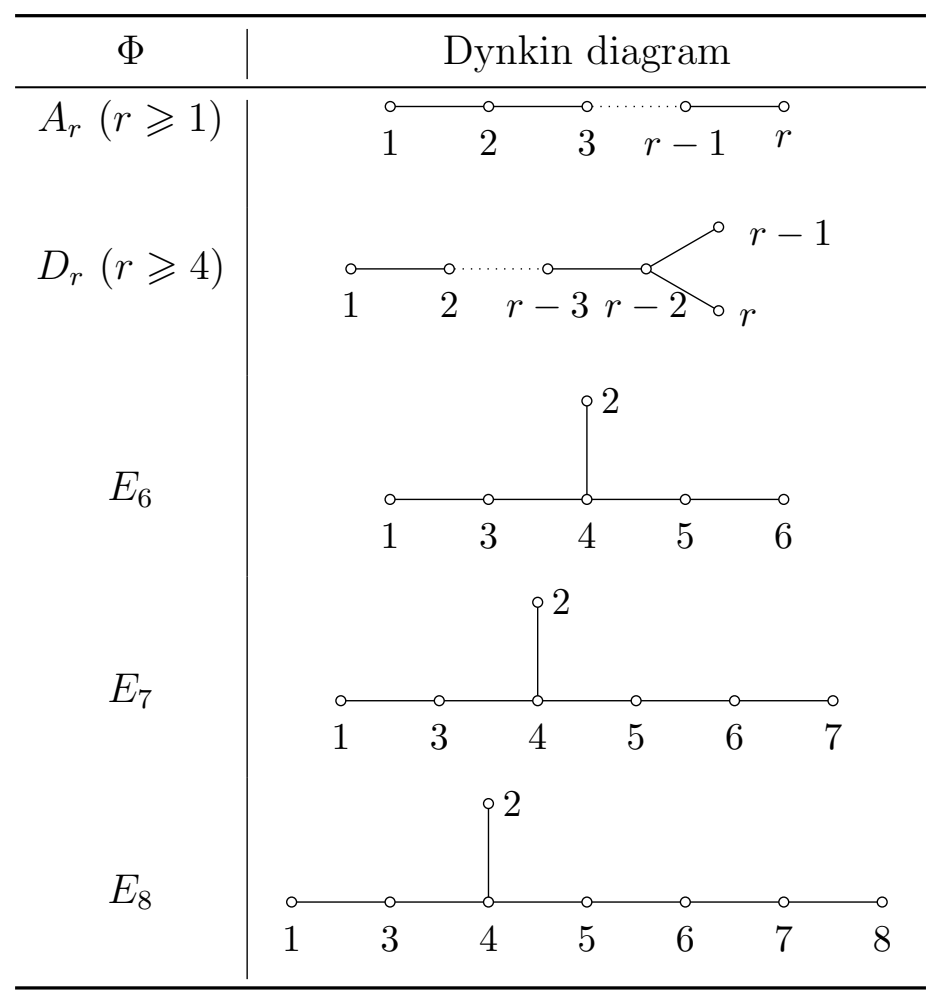

Table 1: Dynkin diagrams of type $A, D, E$.

algebras of type $A, D$, or $E$. In what follows, we fix an ordering on the simple roots as in Table 1; our conventions agree with that in the standard textbook of Humphreys [Hum78].

In order to simplify the notation, we define a distance $d(A, B)$ of two subsets $A, B \subset[r]$ to be

$$
d(A, B):=\min \{d(a, b) \mid a \in A, b \in B\} .
$$

Here, $d(a, b)$ for $a, b \in[r]$ is the minimal distance of elements $a, b$ in the corresponding Dynkin diagram. For example, suppose that $G$ is of type $A_{5}$. Then we have the following enumerations.

$$
d(\{1,2,3\},\{4,5\})=1, \quad d(\{1,2\},\{2\})=0 .
$$

Moreover, when $G$ is of type $D_{4}$, we have $d(\{1,2\},\{4\})=1$ and $d(\{3\},\{4\})=2$.

Definition 12. Let $\mathbf{i}=\left(i_{1}, i_{2}, \ldots, i_{n}\right) \in[r]^{n}$. We say that $\mathbf{i}$ is a jumping walk if

$$
d\left(i_{j},\left\{i_{1}, \ldots, i_{j-1}\right\}\right)=1
$$

for all $2 \leqslant j \leqslant n$.

Example 13. 1. In Type $A$, the words $(1,2,3,4),(3,2,1,4,5)$, and $(3,2,4,1,5)$ are all jumping walks. See Figures 2.1(a), 2.1(b), 2.1(c). 


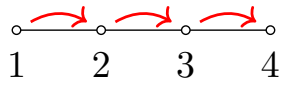

(a) $(1,2,3,4)$.

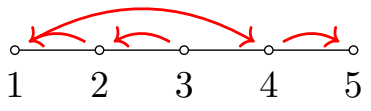

(b) $(3,2,1,4,5)$.

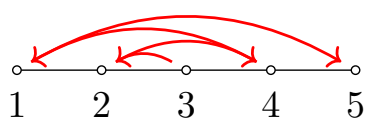

(c) $(3,2,4,1,5)$.

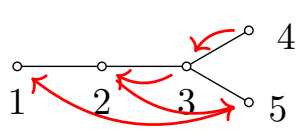

(d) $(4,3,2,5,1)$.

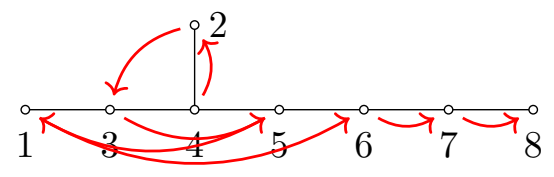

(e) $(4,2,3,5,1,6,7,8)$.

Figure 3.1: Jumping walks.

2. In Type $D,(r-1, r-2, r-3, r, r-4)$ is a jumping walk. See Figure 2.1(d) for $r=5$.

3. In Type $E_{8},(4,2,3,5,1,6,7,8)$ is a jumping walk. See Figure 2.1(e).

Because of the definition, a jumping walk is minimal. More precisely, the indices $\left\{i_{1}, \ldots, i_{n}\right\}$ are all distinct, i.e., the jumping walk visits any given vertex of the Dynkin diagram at most once.

In what follows, we also find it useful to consider words which are 'almost' jumping walks, except that the word begins with a repetition (thus disqualifying it from being a walk), i.e., the initial index appears twice.

Definition 14. Let $\mathbf{i}=\left(i_{0}, i_{1}, \ldots, i_{n}\right) \in[r]^{n+1}$. We say that $\mathbf{i}$ is a hesitant jumping walk if

- $n \geqslant 1$,

- $i_{0}=i_{1}$, and

- the subword $\left(i_{1}, \ldots, i_{n}\right)$ is a jumping walk.

In other words, except for the 'hesitation' at the first step, the remainder of the word is a jumping walk. We refer to the subword $\left(i_{1}, \ldots, i_{n}\right)$ as the jumping component of the hesitant jumping walk.

Definition 15. Let $\boldsymbol{\ell}=\left(\ell_{0}, \ell_{1}, \ldots, \ell_{n}\right) \in \mathbb{Z}_{\geqslant 0}^{n+1}$ and $\mathbf{i} \in[r]^{n+1}$. We say that $\mathbf{i}$ is a hesitant jumping $\boldsymbol{\ell}$-walk if

- $\mathbf{i}$ is a hesitant jumping walk, and

- $\ell_{0}-\ell_{1}<\ell_{1}+\ell_{2}+\cdots+\ell_{n}$.

A word $\mathbf{i}$ is hesitant-jumping- $\boldsymbol{\ell}$-walk-avoiding if there is no subword $\mathbf{j}=\left(i_{j_{0}}, i_{j_{1}}, \ldots, i_{j_{s}}\right)$ of $\mathbf{i}$ which is a hesitant jumping $\left(\ell_{j_{0}}, \ell_{j_{1}}, \ldots, \ell_{j_{s}}\right)$-walk. 
Example 16. Let $G=\operatorname{SL}(3, \mathbb{C})$. Suppose that $\mathbf{i}=(1,2,1)$ and $\left(\ell_{1}, \ell_{2}, \ell_{3}\right) \in \mathbb{Z}_{\geqslant 0}^{3}$. Then the indices $\left(j_{0}, j_{1}\right)=(1,3)$ define a hesitant jumping walk

$$
\left(i_{j_{0}}, i_{j_{1}}\right)=\left(i_{1}, i_{3}\right)=(1,1)
$$

Consequently, the word $\mathbf{i}$ is hesitant-jumping- $\boldsymbol{\ell}$-walk-avoiding if and only if the integers $\ell_{1}, \ell_{2}, \ell_{3}$ satisfy $\ell_{1}-\ell_{3} \geqslant \ell_{3}$.

Example 17. Let $G=\operatorname{SL}(4, \mathbb{C})$. Suppose that $\mathbf{i}=(1,2,1,3,2,1)$ and $\left(\ell_{1}, \ldots, \ell_{6}\right) \in \mathbb{Z}_{\geqslant 0}^{6}$. Then there are five hesitant jumping subwords of $\mathbf{i}$.

\begin{tabular}{l|cccccc}
$\left(j_{0}, j_{1}, \ldots, j_{s}\right)$ & 1 & 2 & 1 & 3 & 2 & 1 \\
\hline$(1,3)$ & 1 & & 1 & & & \\
$(1,3,5)$ & 1 & & 1 & & 2 & \\
$(2,5)$ & & 2 & & & 2 & \\
$(2,5,6)$ & & 2 & & & 2 & 1 \\
$(3,6)$ & & & 1 & & & 1
\end{tabular}

Consequently, the word $\mathbf{i}$ is hesitant-jumping- $\boldsymbol{\ell}$-walk-avoiding if and only if the integers $\ell_{1}, \ldots, \ell_{6}$ satisfy

$$
\begin{aligned}
& \ell_{1}-\ell_{3} \geqslant \ell_{3}, \quad \ell_{1}-\ell_{3} \geqslant \ell_{3}+\ell_{5}, \\
& \ell_{2}-\ell_{5} \geqslant \ell_{5}, \quad \ell_{2}-\ell_{5} \geqslant \ell_{5}+\ell_{6}, \quad \ell_{3}-\ell_{6} \geqslant \ell_{6} .
\end{aligned}
$$

Given the terminology introduced above we state our main theorem.

Theorem 18. Let $G$ be a complex simply-laced semisimple algebraic group of rank $r$. Let $\mathbf{i}=\left(i_{1}, i_{2}, \ldots, i_{n}\right) \in[r]^{n}$ be a word and let $\boldsymbol{\ell}=\left(\ell_{1}, \ldots, \ell_{n}\right) \in \mathbb{Z}_{\geqslant 0}^{n}$. Let $\mathbf{c}=\left\{c_{j, k}\right\}$ be determined from $\mathbf{i}$ as in (1.2). Then the corresponding Grossberg-Karshon twisted cube $(C(\mathbf{c}, \boldsymbol{\ell}), \rho)$ is untwisted if and only if $\mathbf{i}$ is hesitant-jumping- $\boldsymbol{\ell}$-walk-avoiding.

Example 19. Let $G=\mathrm{SL}(3, \mathbb{C})$ and $\mathbf{i}=(1,2,1)$. Then by Example 7 , the subset $C(\mathbf{c}, \ell) \subset \mathbb{R}^{3}$ consists of points $\left(x_{1}, x_{2}, x_{3}\right)$ satisfying:

$$
\begin{array}{rll}
\ell_{1}+x_{2}-2 x_{3}<x_{1}<0 & \text { or } & 0 \leqslant x_{1} \leqslant \ell_{1}+x_{2}-2 x_{3} \\
\ell_{2}+x_{3}<x_{2}<0 & \text { or } & 0 \leqslant x_{2} \leqslant \ell_{2}+x_{3}, \\
\ell_{3}<x_{3}<0 & \text { or } & 0 \leqslant x_{3} \leqslant \ell_{3} .
\end{array}
$$

By Example 16, the word $\mathbf{i}$ is hesitant-jumping- $\boldsymbol{\ell}$-walk-avoiding if and only if

$$
\ell_{1}-\ell_{3} \geqslant \ell_{3}
$$

In Figure 2.2, we draw the twisted cubes for $\left(\ell_{1}, \ell_{2}, \ell_{3}\right)=(3,1,1)$ and $(2,1,2)$, and the former one gives an untwisted twisted cube but not the latter. We present the lattice points in twisted cubes with plus sign in red color and with minus sign in blue color. 


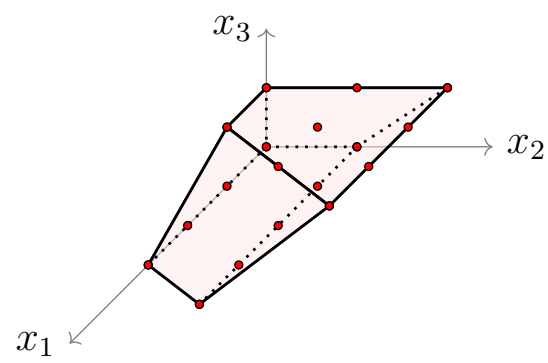

(a) $C(\mathbf{c},(3,1,1))$.

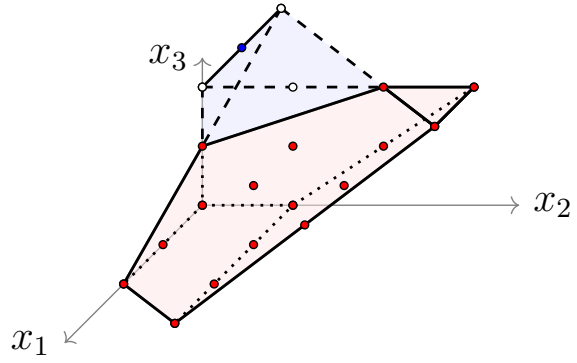

(b) $C(\mathbf{c},(2,1,2))$.

Figure 3.2: Twisted cubes for $G=\mathrm{SL}(3, \mathbb{C})$ and $\mathbf{i}=(1,2,1)$.

We now present a corollary of Theorem 18 which is already observed by Harada and the author [HL15]. In order to state the corollary, it is useful to introduce some terminology in the paper [HL15]. We call a word $\mathbf{i}=\left(i_{1}, \ldots, i_{n}\right)$ a diagram walk if $d\left(i_{j}, i_{j+1}\right)=1$ for all $1 \leqslant j<n$. For a dominant weight $\lambda=\sum_{i=1}^{r} \lambda_{i} \varpi_{i}$, we say $\mathbf{i}=\left(i_{0}, i_{1}, \ldots, i_{n}\right)$ is a hesitant $\lambda$-walk if $i_{0}=i_{1}$, the subword $\left(i_{1}, \ldots, i_{n}\right)$ is a diagram walk, and $\lambda_{i_{n}}>0$. Lastly, we say $\mathbf{i}$ is hesitant- $\lambda$-walk-avoiding if there is no subword which is a hesitant $\lambda$-walk. With these expressions, we present the following corollary of Theorem 18 whose proof is different from that of [HL15].

Corollary 20 (see [HL15, Theorem 2.9]). Let $G$ be a complex simply-laced semisimple algebraic group of rank $r$. Let $\mathbf{i}=\left(i_{1}, i_{2}, \ldots, i_{n}\right) \in[r]^{n}$ be a word and let $\lambda$ be a dominant weight. Let $\mathbf{c}=\left\{c_{j, k}\right\}$ be determined from $\mathbf{i}$ as in (1.2) and let $\boldsymbol{\ell}=\left(\ell_{1}, \ldots, \ell_{n}\right)$ be given by Lemma 11. Then the corresponding Grossberg-Karshon twisted cube $(C(\mathbf{c}, \ell), \rho)$ is untwisted if and only if $\mathbf{i}$ is hesitant- $\lambda$-walk-avoiding.

Proof. By Theorem 18, it is enough to show that the word $\mathbf{i}$ is hesitant-jumping- $\boldsymbol{\ell}$-walkavoiding if and only if it is hesitant- $\lambda$-walk-avoiding. We prove the contrapositive of the claim, that is, we will prove that $\mathbf{i}$ has a subword $\mathbf{j}=\left(i_{j_{0}}, i_{j_{1}}, \ldots, i_{j_{s}}\right)$ which is a hesitant jumping $\left(\ell_{j_{0}}, \ell_{j_{1}}, \ldots, \ell_{j_{s}}\right)$-walk if and only if it has a subword which is a hesitant $\lambda$-walk.

We now suppose that $\mathbf{i}$ has a subword $\mathbf{j}=\left(i_{j_{0}}, i_{j_{1}}, \ldots, i_{j_{s}}\right)$ which is a hesitant $\lambda$-walk. It is clear from the definition that there exists a subword $\mathbf{k}=\left(i_{k_{0}}, i_{k_{1}}, \ldots, i_{k_{p}}\right)$ of $\mathbf{j}$ (i.e., $\left.\left\{k_{0}, k_{1}, \ldots, k_{p}\right\} \subset\left\{j_{0}, j_{1}, \ldots, j_{s}\right\}\right)$ satisfying that $\mathbf{k}$ is a hesitant $\lambda$-walk, $\left\{i_{k_{1}}, \ldots, i_{k_{p}}\right\}$ are all distinct, and $\lambda_{i_{k_{1}}}=\cdots=\lambda_{i_{k_{p}}}=0$. Such a hesitant $\lambda$-walk is called minimal (see [HL15, Definition 4.1]). Accordingly, we may assume that the subword $\mathbf{j}$ is minimal. Since $\left\{i_{j_{1}}, \ldots, i_{j_{s}}\right\}$ are all distinct, $\mathbf{j}$ is a hesitant jumping walk. Moreover, the condition $i_{j_{0}}=i_{j_{1}}$ implies that $\ell_{j_{0}}=\ell_{j_{1}}$ by Lemma 11, and the condition $\lambda_{i_{j_{s}}}>0$ indicates that $\ell_{j_{s}}=\lambda_{i_{j_{s}}}>0$. Therefore we get

$$
\ell_{j_{0}}-\ell_{j_{1}}=0<\ell_{j_{1}}+\cdots+\ell_{j_{s}}
$$

which provides that $\mathbf{j}$ is a hesitant jumping $\left(\ell_{j_{0}}, \ell_{j_{1}}, \ldots, \ell_{j_{s}}\right)$-walk. 
On the other hand, suppose that $\mathbf{i}$ has a subword $\mathbf{j}=\left(i_{j_{0}}, i_{j_{1}}, \ldots, i_{j_{s}}\right)$ which is a hesitant jumping $\left(\ell_{j_{0}}, \ell_{j_{1}}, \ldots, \ell_{j_{s}}\right)$-walk. Then, the condition $i_{j_{0}}=i_{j_{1}}$ provides $\ell_{j_{0}}=\ell_{j_{1}}$, and we get the inequality

$$
0=\ell_{j_{0}}-\ell_{j_{1}}<\ell_{j_{1}}+\cdots+\ell_{j_{s}}
$$

Accordingly, there exists $t \in[s]$ such that $\ell_{j_{t}}>0$. By the definition of jumping walks, one can always find a subword of $\mathbf{j}$ which is a hesitant $\lambda$-walk starting with $i_{j_{0}}, i_{j_{1}}$ and ending at $i_{j_{t}}$. This proves that $\mathbf{i}$ has a subword which is a hesitant $\lambda$-walk, so the result follows.

We enclose this section presenting one more application of Theorem 18 on the NewtonOkounkov body theory. Let $\mathbf{i}=\left(i_{1}, \ldots, i_{n}\right) \in[r]^{n}$ and $\mathbf{m}=\left(m_{1}, \ldots, m_{n}\right) \in \mathbb{Z}_{\geqslant 0}^{n}$. Let $Z_{\mathbf{i}}$ and $\mathcal{L}_{\mathbf{i}, \mathbf{m}}$ be the Bott-Samelson variety and the line bundle on it given by $\mathbf{i}$ and $\mathbf{m}$, respectively. Suppose that $\mathbf{c}=\left\{c_{j, k}\right\}$ and $\boldsymbol{\ell}$ are determined from (1.2) and (1.3), and $C(\mathbf{c}, \boldsymbol{\ell})$ is the corresponding twisted cube. Harada and Yang [HY16] constructed a valuation $\nu: H^{0}\left(Z_{\mathbf{i}}, \mathcal{L}_{\mathbf{i}, \mathbf{m}}^{\otimes k}\right) \backslash\{0\} \rightarrow \mathbb{Z}^{n}$ for each $k \geqslant 0$ such that if the corresponding twisted cube $C(\mathbf{c}, \ell)$ is untwisted then it coincides with the Newton-Okounkov body $\Delta\left(Z_{\mathbf{i}}, \mathcal{L}_{\mathbf{i}, \mathbf{m}}, \nu\right)$ defined to be

$$
\Delta\left(Z_{\mathbf{i}}, \mathcal{L}_{\mathbf{i}, \mathbf{m}}, \nu\right)=\overline{\operatorname{conv}\left(\bigcup_{k>0}\left\{\frac{\nu(\sigma)}{k} \mid \sigma \in H^{0}\left(Z_{\mathbf{i}}, \mathcal{L}_{\mathbf{i}, \mathbf{m}}^{\otimes k}\right) \backslash\{0\}\right\}\right)}
$$

up to a certain coordinate change (see [HY16, Theorem 3.4] for more details). As a consequence, we get the following corollary which is related to the question (2) in [HL15, Section 5].

Corollary 21. Let $G$ be a complex simply-laced semisimple algebraic group of rank $r$. Let $\mathbf{i}=\left(i_{1}, \ldots, i_{n}\right) \in[r]^{n}$ be a word and let $\mathbf{m}=\left(m_{1}, \ldots, m_{n}\right) \in \mathbb{Z}_{\geqslant 0}^{n}$. Let $\mathbf{c}=\left\{c_{j, k}\right\}$ and $\boldsymbol{\ell} \in \mathbb{Z}^{n}$ be determined from $\mathbf{i}$ and $\mathbf{m}$ as in (1.2) and (1.3). If the word $\mathbf{i}$ is hesitant-jumping$\boldsymbol{\ell}$-walk-avoiding, then the twisted cube $C(\mathbf{c}, \boldsymbol{\ell})$ coincides with the Newton-Okounkov body $\Delta\left(Z_{\mathbf{i}}, \mathcal{L}_{\mathbf{i}, \mathbf{m}}, \nu\right)$ up to a coordinate change, where $\nu$ is the valuation constructed in [HY16].

\section{Proof of the main theorem}

In this section, we will present a proof of the main theorem. We start with a proposition which will be used in the proof. One can see that the subsequent proposition holds only in simply-laced cases.

Proposition 22. Let $G$ be a complex simply-laced semisimple algebraic group of rank $r$. Let $\mathbf{i}=\left(i_{1}, i_{2}, \ldots, i_{n}\right) \in[r]^{n}$ be a word, and let $\mathbf{c}=\left\{c_{j, k}\right\}$ be determined from $\mathbf{i}$ as in $(1.2)$. Suppose that $\left(j_{0}<j_{1}<j_{2}<\cdots<j_{s}\right)$ is an increasing sequence of elements in $[n]$, and $\mathbf{j}=\left(i_{j_{0}}, i_{j_{1}}, \ldots, i_{j_{s}}\right)$ is the corresponding subword. Then, the sequence $\left(j_{0}<j_{1}<j_{2}<\right.$ $\left.\cdots<j_{s}\right)$ satisfies the condition

$$
\begin{aligned}
& c_{j_{0}, j_{1}}=2, \\
& c_{j_{1}, j_{t}}+c_{j_{2}, j_{t}}+\cdots+c_{j_{t-1}, j_{t}}=-1 \quad \text { for } 2 \leqslant t \leqslant s
\end{aligned}
$$


if and only if $\mathbf{j}$ is a hesitant jumping walk.

Lemma 23. Suppose that $G$ is simply-laced and $\left(i_{1}, \ldots, i_{n-1}\right)$ is a jumping walk. For $i_{n} \in[r]$, we have the following.

1. If $d\left(i_{n},\left\{i_{1}, \ldots, i_{n-1}\right\}\right)=0$, then $c_{i_{n}, i_{1}}+\cdots+c_{i_{n}, i_{n-1}} \geqslant 0$.

2. If $d\left(i_{n},\left\{i_{1}, \ldots, i_{n-1}\right\}\right)=1$, then $c_{i_{n}, i_{1}}+\cdots+c_{i_{n}, i_{n-1}}=-1$.

3. If $d\left(i_{n},\left\{i_{1}, \ldots, i_{n-1}\right\}\right)>1$, then $c_{i_{n}, i_{1}}+\cdots+c_{i_{n}, i_{n-1}}=0$.

Here, $\boldsymbol{c}_{j, k}$ are Cartan integers:

$$
c_{j, k}=\left\langle\alpha_{j}, \alpha_{k}^{\vee}\right\rangle= \begin{cases}2 & \text { if } j=k, \\ -1 & \text { if } d(j, k)=1 \\ 0 & \text { otherwise. }\end{cases}
$$

Proof. Note that since $\left(i_{1}, \ldots, i_{n-1}\right)$ is a jumping walk, the set $I:=\left\{i_{1}, \ldots, i_{n-1}\right\}$ forms an interval on the Dynkin diagram, i.e., if $j \in[n]$ satisfies $\min I<j<\max I$, then $j \in I$.

We consider the first case. Since the distance between $i_{n}$ and the set $I$ is zero, $i_{n} \in I$. Suppose that $i_{n}$ sits in the right-most/left-most position among $I$, i.e., $i_{n}=\min I$ or $i_{n}=\max I$. Then,

$$
c_{i_{n}, i_{1}}+\cdots+c_{i_{n}, i_{n-1}}=2-1=1 \geqslant 0,
$$

which is the desired inequality. Suppose that $i_{n}$ is neither maximum nor minimum of $I$. Then, we have that

$$
c_{i_{n}, i_{1}}+\cdots+c_{i_{n}, i_{n-1}}=2-1-1=0 \geqslant 0 .
$$

Consequently, we prove the claim for (1). For the second and the third cases, by the definition of Cartan integers, we get the required equalities.

Proof of Proposition 22. Suppose that a subword $\mathbf{j}=\left(i_{j_{0}}, i_{j_{1}}, \ldots, i_{j_{s}}\right)$ of $\mathbf{i}$ is a hesitant jumping walk. Then, by the hesitant condition $i_{j_{0}}=i_{j_{1}}$, we have $c_{j_{0}, j_{1}}=2$. Moreover, the jumping walk condition $d\left(i_{j_{t}},\left\{i_{j_{1}}, \ldots, i_{j_{t-1}}\right\}\right)=1$ and Lemma 23 imply that

$$
c_{j_{1}, j_{t}}+\cdots+c_{j_{t-1}, j_{t}}=c_{i_{j_{t}}, i_{j_{1}}}+\cdots+c_{i_{j_{t}}, i_{j_{t-1}}}=-1
$$

which proves the "if" part of the proposition.

Suppose that we have an increasing sequence $\left(j_{0}<j_{1}<\cdots<j_{s}\right)$ satisfying conditions. Consider the subword $\mathbf{j}=\left(i_{j_{0}}, i_{j_{1}}, \ldots, i_{j_{s}}\right)$. The first condition $c_{j_{0}, j_{1}}=2$ implies that $i_{j_{0}}=i_{j_{1}}$. Accordingly, the word $\mathbf{j}$ hesitates at the first. When $t=2$, the second condition becomes $c_{j_{1}, j_{2}}=-1$. Then by $(3.1)$, we have that $d\left(i_{j_{2}}, i_{j_{1}}\right)=1$, so that $\left(i_{j_{0}}, i_{j_{1}}, i_{j_{2}}\right)$ is a hesitant jumping walk. Using an induction on $s$ and Lemma 23, we prove the "only if" part of the proposition. 


\subsection{Necessity}

We first prove that if $\mathbf{i}$ has a subword $\mathbf{j}=\left(i_{j_{0}}, i_{j_{1}}, \ldots, i_{j_{s}}\right)$ which is a hesitant jumping $\left(\ell_{j_{0}}, \ell_{j_{1}}, \ldots, \ell_{j_{s}}\right)$-walk, then the corresponding twisted cube is twisted. Suppose that $\mathbf{j}=$ $\left(i_{j_{0}}, i_{j_{1}}, \ldots, i_{j_{s}}\right)$ is a subword of $\mathbf{i}$ which is a hesitant jumping $\left(\ell_{j_{0}}, \ell_{j_{1}}, \ldots, \ell_{j_{s}}\right)$-walk, so we have

$$
\ell_{j_{0}}-\ell_{j_{1}}<\ell_{j_{1}}+\cdots+\ell_{j_{s}}
$$

Then by Proposition 22, the integers $\left\{c_{j, k}\right\}$ satisfy that

$$
\begin{aligned}
& c_{j_{0}, j_{1}}=2 \text {, } \\
& c_{j_{1}, j_{t}}+c_{j_{2}, j_{t}}+\cdots+c_{j_{t-1}, j_{t}}=-1 \quad \text { for } 2 \leqslant t \leqslant s .
\end{aligned}
$$

We then wish to show that $(C(\mathbf{c}, \boldsymbol{\ell}), \rho)$ is twisted. To prove that $(C(\mathbf{c}, \ell), \rho)$ is twisted, it is enough to find an element $\sigma$ of $\{+,-\}^{n}$ and an index $k \in[n]$ such that $m_{\sigma, k}<0$. To achieve this, we consider the element $\sigma=\left(\sigma_{1}, \ldots, \sigma_{n}\right) \in\{+,-\}^{n}$ given by

$$
\sigma_{p}= \begin{cases}- & \text { if } p \in\left\{j_{0}, j_{1}, \ldots, j_{s}\right\} \\ + & \text { otherwise }\end{cases}
$$

By the definition of $m_{\sigma}$ and $m_{\sigma, p}=0$ for $p \notin\left\{j_{0}, j_{1}, \ldots, j_{s}\right\}$, we then have

$$
m_{\sigma, j_{t}}=\ell_{j_{t}}-\sum_{p \in\left\{j_{t+1}, \ldots, j_{s}\right\}} c_{j_{t}, p} m_{\sigma, p} \quad \text { for } 0 \leqslant t \leqslant s .
$$

We know that $c_{j_{0}, j_{1}}=\left\langle\alpha_{i_{j_{0}}}, \alpha_{i_{j_{1}}}^{\vee}\right\rangle=2$ if and only if $i_{j_{0}}=i_{j_{1}}$. Moreover, in this case we have $c_{j_{0}, p}=c_{j_{1}, p}$ for all $p$. From these considerations, we have:

$$
\begin{aligned}
m_{\sigma, j_{0}} & =\ell_{j_{0}}-\sum_{p \in\left\{j_{1}, \ldots, j_{s}\right\}} c_{j_{0}, p} m_{\sigma, p} \\
& =\ell_{j_{0}}-c_{j_{0}, j_{1}} m_{\sigma, j_{1}}-\sum_{p \in\left\{j_{2}, \ldots, j_{s}\right\}} c_{j_{0}, p} m_{\sigma, p} \\
& =\ell_{j_{0}}-2\left(\ell_{j_{1}}-\sum_{p \in\left\{j_{2}, \ldots, j_{s}\right\}} c_{j_{1}, p} m_{\sigma, p}\right)-\sum_{p \in\left\{j_{2}, \ldots, j_{s}\right\}} c_{j_{0}, p} m_{\sigma, p} \\
& =\ell_{j_{0}}-2 \ell_{j_{1}}+\sum_{p \in\left\{j_{2}, \ldots, j_{s}\right\}} c_{j_{1}, p} m_{\sigma, p} \\
& =\ell_{j_{0}}-\ell_{j_{1}}-m_{\sigma, j_{1}} .
\end{aligned}
$$

We now claim that $m_{\sigma, j_{0}}<0$; as already noted, this suffices to prove the necessity of the theorem. In order to prove this claim, it is enough to show that

$$
m_{\sigma, j_{1}}=\ell_{j_{1}}+\cdots+\ell_{j_{s}}
$$


This is because if (3.5) holds, then we get

$$
\begin{aligned}
m_{\sigma, j_{0}} & =\ell_{j_{0}}-\ell_{j_{1}}-m_{\sigma, j_{1}} \\
& =\ell_{j_{0}}-\ell_{j_{1}}-\left(\ell_{j_{1}}+\cdots+\ell_{j_{s}}\right) \\
& <0 \quad(\text { by }(3.2))
\end{aligned}
$$

which proves the claim, so now we prove (3.5). Using (3.3) and the description (3.4) of $m_{\sigma, j_{t}}$, we have that

$$
\begin{aligned}
m_{\sigma, j_{1}} & =\ell_{j_{1}}-c_{j_{1}, j_{2}} m_{\sigma, j_{2}}-\cdots-c_{j_{1}, j_{s}} m_{\sigma, j_{s}} \\
& =\ell_{j_{1}}+\left(\ell_{j_{2}}-c_{j_{2}, j_{3}} m_{\sigma, j_{3}}-\cdots-c_{j_{2}, j_{s}} m_{\sigma, j_{s}}\right)-c_{j_{1}, j_{3}} m_{\sigma, j_{3}}-\cdots-c_{j_{1}, j_{s}} m_{\sigma, j_{s}} \\
& =\ell_{j_{1}}+\ell_{j_{2}}-\left(c_{j_{2}, j_{3}}+c_{j_{1}, j_{3}}\right) m_{\sigma, j_{3}}-\cdots-\left(c_{j_{2}, j_{s}}+c_{j_{1}, j_{s}}\right) m_{\sigma, j_{s}} \\
& =\ell_{j_{1}}+\ell_{j_{2}}+\left(\ell_{j_{3}}-c_{j_{3}, j_{4}} m_{\sigma, j_{4}}-\cdots-c_{j_{3}, j_{s}} m_{\sigma, j_{s}}\right)-\cdots-\left(c_{j_{2}, j_{s}}+c_{j_{1}, j_{s}}\right) m_{\sigma, j_{s}} \\
& =\ell_{j_{1}}+\ell_{j_{2}}+\ell_{j_{3}}-\left(c_{j_{3}, j_{4}}+c_{j_{2}, j_{4}}+c_{j_{1}, j_{4}}\right) m_{\sigma, j_{4}}-\cdots-\left(c_{j_{3}, j_{s}}+c_{j_{2}, j_{s}}+c_{j_{1}, j_{s}}\right) m_{\sigma, j_{s}} \\
& =\cdots=\ell_{j_{1}}+\ell_{j_{2}}+\cdots+\ell_{j_{s-1}}-\left(c_{j_{s-1}, j_{s}}+\cdots+c_{j_{1}, j_{s}}\right) m_{\sigma, j_{s}} \\
& =\ell_{j_{1}}+\ell_{j_{2}}+\cdots+\ell_{j_{s}} .
\end{aligned}
$$

Consequently, we prove the equation (3.5), so the necessity of the theorem follows.

\subsection{Sufficiency}

We now prove that the twistedness implies the existence of a subword $\mathbf{j}=\left(i_{j_{0}}, i_{j_{1}}, \ldots, i_{j_{s}}\right)$ which is a hesitant jumping $\left(\ell_{j_{0}}, \ell_{j_{1}}, \ldots, \ell_{j_{s}}\right)$-walk. To give a proof, we prepare one lemma.

Lemma 24. Suppose that a sequence $\left(j_{1}<j_{2}<\cdots<j_{s-1}\right)$ of indices defines a jumping walk $\left(i_{j_{1}}, \ldots, i_{j_{s-1}}\right)$. If for some $j_{s}>j_{s-1}$ we have

$$
c_{j_{1}, j_{s}}+c_{j_{2}, j_{s}}+\cdots+c_{j_{s-1}, j_{s}}<0
$$

then the sequence $\left(j_{1}<j_{2}<\cdots<j_{s-1}<j_{s}\right)$ also defines a jumping walk and $c_{j_{1}, j_{s}}+$ $c_{j_{2}, j_{s}}+\cdots+c_{j_{s-1}, j_{s}}=-1$.

Proof. Assume on the contrary that $\left(j_{1}<j_{2}<\cdots<j_{s-1}<j_{s}\right)$ does not define a jumping walk, i.e., $d\left(i_{j_{s}},\left\{i_{j_{1}}, \ldots, i_{j_{s-1}}\right\}\right) \neq 1$. Then, by Lemma 23 , we have that

$$
c_{j_{1}, j_{s}}+c_{j_{2}, j_{s}}+\cdots+c_{j_{s-1}, j_{s}}=c_{i_{s}, i_{j_{1}}}+c_{i_{s}, i_{j_{2}}}+\cdots+c_{i_{s}, i_{j_{s-1}}} \geqslant 0,
$$

which contradicts to the assumption. As a consequence, we prove the lemma.

By Theorem 5, there exists an element $\sigma$ of $\{+,-\}^{n}$ and an index $k$ such that $m_{\sigma, k}<0$. For such a choice of $\sigma$, we may assume without loss of generality that $k$ is chosen to be the maximal such index, i.e., $m_{\sigma, k}<0$ and $m_{\sigma, s} \geqslant 0$ for $s>k$. Recall that

$$
m_{\sigma, k}=\ell_{k}-\sum_{s>k} c_{k, s} m_{\sigma, s}
$$


By assumption $m_{\sigma, k}<0$, we have that

$$
\sum_{s>k} c_{k, s} m_{\sigma, s}>\ell_{k} \geqslant 0
$$

Since $m_{\sigma, s} \geqslant 0$ for $s>k$, this implies that there exists some $p>k$ with $c_{k, p}>0$ and $m_{\sigma, p}>0$. Choose $j_{1}$ to be the minimal such index. Consequently, $c_{k, s} \leqslant 0$ or $m_{\sigma, s}=0$ for all $k<s<j_{1}$, so we have that

$$
\ell_{k}<\sum_{s>k} c_{k, s} m_{\sigma, s} \leqslant c_{k, j_{1}} m_{\sigma, j_{1}}+\sum_{s>j_{1}} c_{k, s} m_{\sigma, s}
$$

By definition, we have that $c_{k, j_{1}}=\left\langle\alpha_{i_{j_{1}}}, \alpha_{i_{k}}^{\vee}\right\rangle>0$ if and only if $i_{k}=i_{j_{1}}$. Furthermore, in this case we get $c_{k, j_{1}}=2$ and $c_{j_{1}, s}=c_{k, s}$ for all $s$. From these observations, we get:

$$
c_{k, j_{1}} m_{\sigma, j_{1}}+\sum_{s>j_{1}} c_{k, s} m_{\sigma, s}=2\left(\ell_{j_{1}}-\sum_{s>j_{1}} c_{j_{1}, s} m_{\sigma, s}\right)+\sum_{s>j_{1}} c_{j_{1}, s} m_{\sigma, s} .
$$

Combining (3.6) and (3.7), we have that

$$
\ell_{k}-\ell_{j_{1}}<\ell_{j_{1}}-\sum_{s>j_{1}} c_{j_{1}, s} m_{\sigma, s}=m_{\sigma, j_{1}}
$$

First suppose $-\sum_{s>j_{1}} c_{j_{1}, s} m_{\sigma, s} \leqslant 0$. In this case, we have that

$$
\ell_{k}-\ell_{j_{1}}<\ell_{j_{1}}
$$

Accordingly, the sequence $\left(j_{0}=k<j_{1}\right)$ satisfies the three required conditions of hesitant jumping $\left(\ell_{j_{0}}, \ell_{j_{1}}\right)$-walks, so we are done.

On the other hand, if $-\sum_{s>j_{1}} c_{j_{1}, s} m_{\sigma, s}>0$, we set $j_{0}=k$ and define $j_{2}$ as follows. Since $m_{\sigma, s} \geqslant 0$ for $s>k$ by assumption, in order for the summand $\sum_{s>j_{1}} c_{j_{1}, s} m_{\sigma, s}$ to be strictly negative there must exist an index $j_{2}>j_{1}$ with $c_{j_{1} j_{2}}<0$ and $m_{\sigma, j_{2}}>0$. Note that since $c_{j_{1} j_{2}}=\left\langle\alpha_{i_{j_{2}}}, \alpha_{i_{j_{1}}}^{\vee}\right\rangle$ we have that $c_{j_{1}, j_{2}}=-1$. Choose $j_{2}$ to be the minimal such index, i.e., $c_{j_{1}, s} \geqslant 0$ or $m_{\sigma, s}=0$ for all $j_{1}<s<j_{2}$. Then we have that

$$
\begin{aligned}
m_{\sigma, j_{1}} & =\ell_{j_{1}}-\sum_{s>j_{1}} c_{j_{1}, s} m_{\sigma, s} \\
& \leqslant \ell_{j_{1}}-c_{j_{1}, j_{2}} m_{\sigma, j_{2}}-\sum_{s>j_{2}} c_{j_{1}, s} m_{\sigma, s} \\
& =\ell_{j_{1}}+m_{\sigma, j_{2}}-\sum_{s>j_{2}} c_{j_{1}, s} m_{\sigma, s} \\
& =\ell_{j_{1}}+\ell_{j_{2}}-\sum_{s>j_{2}}\left(c_{j_{2}, s}+c_{j_{1}, s}\right) m_{\sigma, s} .
\end{aligned}
$$


If $-\sum_{s>j_{2}}\left(c_{j_{2}, s}+c_{j_{1}, s}\right) m_{\sigma, s} \leqslant 0$, then the sequence $\left(j_{0}=k<j_{1}<j_{2}\right)$ satisfies the three required conditions since we get the following inequality by considering (3.8) and (3.9):

$$
\ell_{j_{0}}-\ell_{j_{1}}<m_{\sigma, j_{1}} \leqslant \ell_{j_{1}}+\ell_{j_{2}} .
$$

Otherwise, i.e., $\sum_{s>j_{2}}\left(c_{j_{2}, s}+c_{j_{1}, s}\right) m_{\sigma, s}<0$, then we choose $j_{3}>j_{2}$ to be the minimal index such that $c_{j_{2}, s}+c_{j_{1}, s}<0$ and $m_{\sigma, s}>0$. Since $\left(j_{1}<j_{2}\right)$ defines a jumping walk, we have that $\left(j_{1}<j_{2}<j_{3}\right)$ defines a jumping walk and $c_{j_{2}, j_{3}}+c_{j_{1}, j_{3}}=-1$ by Lemma 24 . Therefore the inequality (3.9) becomes to

$$
\begin{aligned}
m_{\sigma, j_{1}} & \leqslant \ell_{j_{1}}+\ell_{j_{2}}+m_{\sigma, j_{3}}-\sum_{s>j_{3}}\left(c_{j_{2}, s}+c_{j_{1}, s}\right) m_{\sigma, s} \\
& =\ell_{j_{1}}+\ell_{j_{2}}+\ell_{j_{3}}-\sum_{s>j_{3}}\left(c_{j_{3}, s}+c_{j_{2}, s}+c_{j_{1}, s}\right) m_{\sigma, s} .
\end{aligned}
$$

If $-\sum_{s>j_{3}}\left(c_{j_{3}, s}+c_{j_{2}, s}+c_{j_{1}, s}\right) m_{\sigma, s} \leqslant 0$, then the sequence $\left(j_{0}=k<j_{1}<j_{2}<j_{3}\right)$ satisfies the three required conditions. Otherwise, we may repeat the above argument as many times as necessary. Since the indices $j_{t}$ are bounded above by $n$, this process must stop, i.e., there must exist some $s \geqslant 1$ such that the sequence $j_{0}<j_{1}<\cdots<j_{s}$ found in this manner satisfies the requirements. Consequently, we prove the sufficiency of the theorem.

\section{Acknowledgements}

The author thanks Professor Megumi Harada for bringing her this question, and thanks Professor Dong Youp Suh for his supports throughout the project.

\section{References}

[FLS] Naoki Fujita, Eunjeong Lee, and Dong Youp Suh. Algebraic and geometric properties of flag Bott-Samelson varieties and applications to representations. arXiv: $1805.01664 \mathrm{v} 3$.

[Fuj18] Naoki Fujita. Newton-Okounkov bodies for Bott-Samelson varieties and string polytopes for generalized Demazure modules. J. Algebra, 515:408-447, 2018. https://doi.org/10.1016/j.jalgebra.2018.08.019.

[GK94] Michael Grossberg and Yael Karshon. Bott towers, complete integrability, and the extended character of representations. Duke Math. J., 76(1):23-58, 1994. https://doi .org/10.1215/S0012-7094-94-07602-3.

[HL15] Megumi Harada and Eunjeong Lee. Grossberg-Karshon twisted cubes and hesitant walk avoidance. Pacific J. Math., 278(1):119-136, 2015. https://doi.org/10.2140/pjm.2015.278.119.

[Hum78] James E. Humphreys. Introduction to Lie algebras and representation theory, volume 9 of Graduate Texts in Mathematics. Springer-Verlag, New York-Berlin, 1978. Second printing, revised. https://doi .org/10.1007/978-1-4612-6398-2. 
[HY15] Megumi Harada and Jihyeon Jessie Yang. Grossberg-Karshon twisted cubes and basepoint-free divisors. J. Korean Math. Soc., 52(4):853-868, 2015. https://doi.org/10.4134/JKMS. 2015.52.4.853.

[HY16] Megumi Harada and Jihyeon Jessie Yang. Newton-Okounkov bodies of BottSamelson varieties and Grossberg-Karshon twisted cubes. Michigan Math. J., 65(2):413-440, 2016. https://doi.org/10.1307/mmj/1465329020.

[Kav15] Kiumars Kaveh. Crystal bases and Newton-Okounkov bodies. Duke Math. J., 164(13):2461-2506, 2015. https://doi .org/10.1215/00127094-3146389.

[Pas10] Boris Pasquier. Vanishing theorem for the cohomology of line bundles on BottSamelson varieties. J. Algebra, 323(10):2834-2847, 2010. https://doi.org/10.1016/j.jalgebra.2010.03.016. 